\title{
SCIATIC NERVE BLOCK COMBINED WITH FEMORAL CATHETER IN POSTOPERATIVE PAIN MANAGEMENT AFTER KNEE MEGAENDOPROSTHESIS IN TWO PATIENTS WITH OSTEOSARCOMA
}

\author{
M. Dobrić, J. Žarko, A. Miletić, T. Beker
}

\section{BACKGROUND AND AIMS}

Total knee arthroplasty (TKA) is associated with severe postoperative pain. The femoral nerve originates from the L2, L3 and L4 nerve roots and innervates the anterior thigh and the knee ${ }^{1,2}$. The sciatic nerve, which originates from the nerve roots from $L 4$ to S3, innervates the posterior thigh and the portion of the leg distal to the knee with exclusion of the medial component ${ }^{3}$. Parenteral opioids are still widely used, being slowly replaced by peripheral nerve blocks and periarticular injections. The ultrasound guided techniques reduce the risk of neurologic complications.

\section{METHODS}

15 yrs old male, BMI 19, with osteosarcoma in left distal femur scheduled for tumour resection and total knee arthroplasty following chemotherapy refused the proposed option of regional continuous neuroaxial anaesthesia. The patient opted for general anaesthesia and peripheral nerve block for postoperative pain control. 21 yrs old female, BMI 17, with right distal femur osteosarcoma and solitary pulmonary metastasis successfully treated with chemotherapy agreed to spinal anaesthesia with intravenous sedation and peripheral nerve blocks for postoperative pain management. In both cases surgical procedure lasted for four hours and went uneventfully. Peripheral nerve blocks were performed in PACU, immediately after the surgical procedure. Ultrasound guided femoral catheter placement for intermittent femoral nerve block with bolus of levobupivacain $0.25 \% 15 \mathrm{~mL}$ was followed by sciatic nerve block guided by peripheral nerve stimulator, with $10 \mathrm{~mL}$ of $0.5 \%$ levobupivacain in both patients.
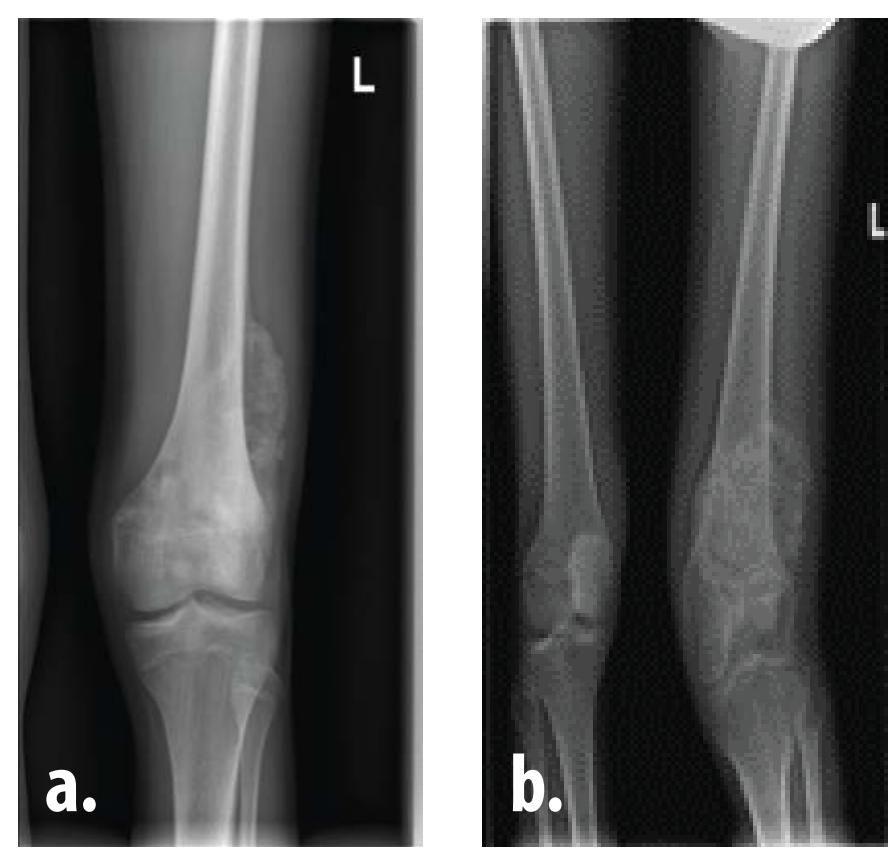

Figure 1. Plain radiograph of left femur with destructive process in distal third, involving the bone and soft tissue, Patient 1. (a) and 2. (b).
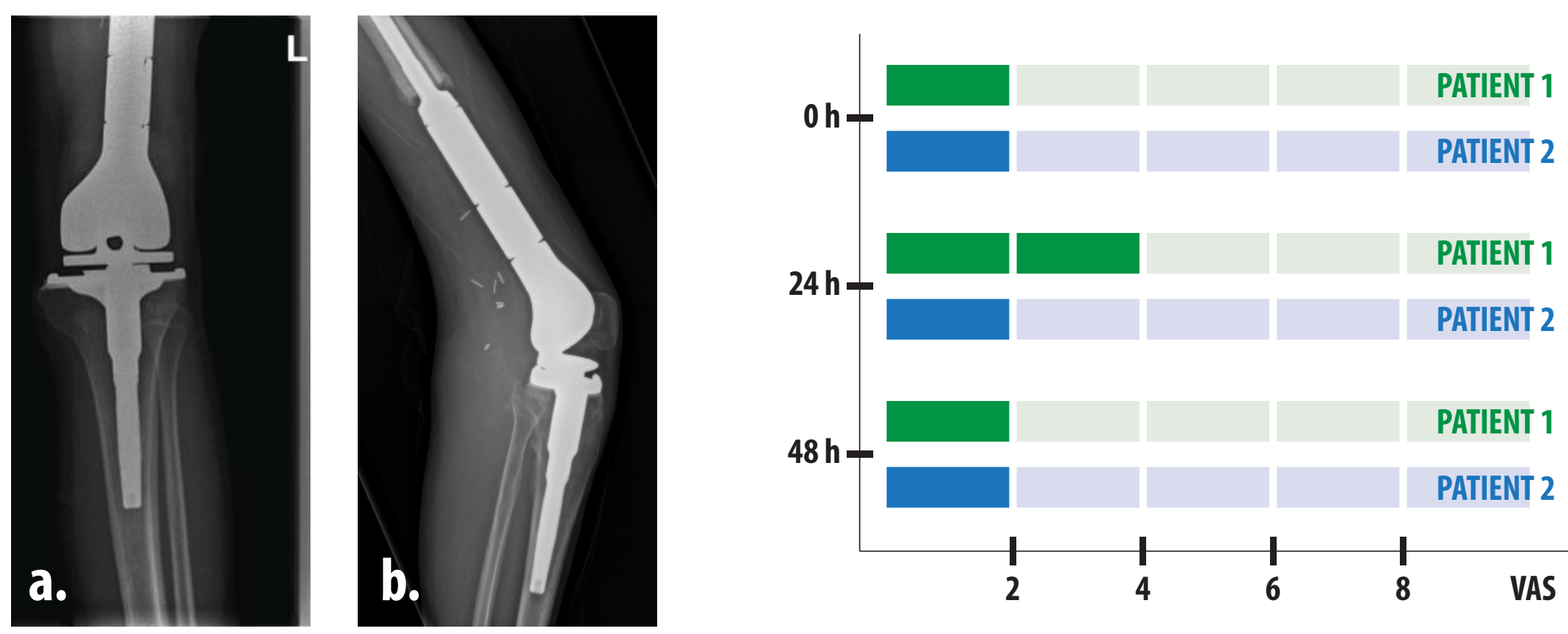

Figure 2. Plain radiograph of left knee following complete tumore resection and total knee alloarthroplastiy with megaprosthesis in Patients 1. (a) and 2 (b).
Figure 3. VAS scores at $0,12,24$ and $48 \mathrm{hrs}$ Patient 1. (OA + PNBS), Patient 2. (SA + PNBS)

\section{RESULTS}

In both cases the patient was sent to the ward with VAS 2 . The maximum VAS was 4 at 24 hours after surgery in patient who underwent general anaesthesia, while the patient who was in spinal did not exceed VAS 2 . Both patients received levobupivacain $0.25 \%$ via femoral catheter, 8 hourly. Early physiotherapy started 24 hours after the surgery in both patients. 48 hours after surgery both patients had VAS 2, and femoral catheters were removed. In addition to boluses via femoral catheter the general anaesthesia patient received altogether one dose of opioid and two doses of NSAIR in the whole postoperative period, while the spinal anaesthesia patient received NSAIR until the day 3.

\section{References:}

1. Muhly WT, Gurnaney HG, Ganesh A. Regional anesthesia for pediatric knee surgery: a review of the indications, procedures, outcomes, safety, and challenges. Local Reg Anesthesia. 2015;8:85-91

2. Shah RD, Suresh S. Applications of regional anaesthesia in paediatrics. Br J Anesth, 2013; 111(1):114-24. doi: 10.1093/bja/aet379.

\section{CONCLUSION}

- Regional anaesthesia provide powerful analgesia with minimal risk and few adverse effects.

- The technique combining continuous femoral with sciatic nerve block proved to be adequate for postoperative pain management after major knee surgery in our two patients.

- Regional anaesthesia provides an effective alternative to reliance on opioids for postoperative pain relief 4 .
3. Gerrard C, Roberts S. Ultrasound-Guided Regional Anaesthesia in the Paediatric Population. ISRN Anesthesiology, 2012; Article ID 169043

4. Sinha SA, Mutha SC, Phalgune DS. Efficacy of Sciatic Nerve Block for Pain Management in below Knee Orthopaedic Surgery. Journal of Clinical and Diagnostic Research, 2016;10(9):UC17-UC20. doi:10.7860/JCDR/2016/20418.8496. 